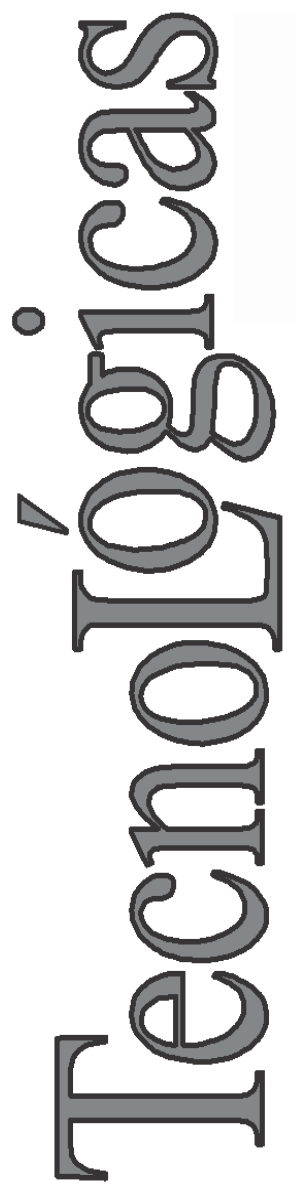

\title{
Plataformas de Laboratorio de Bajo Costo Basadas en el Protocolo ZigBee
}

\section{Low Cost ZigBee Protocol Based Laboratory Platforms}

\author{
Alvaro Romero-Acero ${ }^{1}$ \\ Alejandro Marín-Cano ${ }^{2}$ \\ Eliana I. Arango-Zuluaga ${ }^{3}$
}

1 Departamento de Energía Eléctrica y Automática,

Facultad de Minas, Universidad Nacional de Colombia sede Medellín, Medellín-Colombia alromeroac@unal.edu.co

2 Departamento de Energía Eléctrica y Automática, Facultad de Minas, Universidad Nacional de Colombia sede Medellín, Medellín-Colombia amarincan@unal.edu.co correo

3 Departamento de Energía Eléctrica y Automática, Facultad de Minas, Universidad Nacional de Colombia sede Medellín, Medellín-Colombia eiarangoz@unal.edu.co correo 


\section{Resumen}

Este trabajo presenta una plataforma de comunicación inalámbrica, de bajo costo, basada en el protocolo ZigBee. Se ha diseñado con el propósito de fortalecer el uso de las tecnologías de la información en el aula de clase. Las guías de las prácticas de laboratorio a desarrollar están enfocadas a estudiantes de pregrado en ingeniería para el área de las telecomunicaciones. La estructura de la plataforma se compone de: Prácticas de laboratorio diseñadas a medida, herramientas web, sistema embebido de comunicación inalámbrica con adquisición de datos en tiempo real, e interface gráfica Human Machine Interface (HMI), la cual registra los datos de señales analógicas y digitales.

\section{Palabras clave}

Plataforma de laboratorio; protocolo ZigBee; redes inalámbricas; sistema embebido; educación en ingeniería.

\section{Abstract}

This paper presents a low cost wireless communication platform, based on the ZigBee protocol. It is designed with the purpose to strengthen the use of information technology in the classroom. Guides laboratory practices are focused on developing undergraduate engineering students to the area of telecommunications. The platform structure is composed of: Labs custom designed, web tools embedded wireless communication system for data acquisition in real time, and the Human Machine Interface (HMI), which records analog data and digital.

\section{Keywords}

Human Machine Interface; learning platform; ZigBee protocol; wireless; embedded system. 


\section{INTRODUCCIÓN}

La introducción de las tecnologías de la información y las comunicaciones (TIC) en la educación, ha permitido fomentar el desarrollo de nuevas competencias en los estudiantes y ha aumentado su interés por las actividades de investigación e innovación (Knezek \& Christensen, 2008). En el contexto de la educación superior en Colombia, la falta de infraestructura que facilite el uso de las TIC, tanto en clases teóricas como en sesiones prácticas en el laboratorio, es uno de los aspectos que ha dificultado su utilización (Velásquez \& López, 2008).

Una solución al problema de infraestructura se presenta en el trabajo de Hashemian y Pearson (2009), que propone la creación de laboratorios de acceso remoto a bajo costo, usando los servicios cliente/servidor institucionales. Otra solución se presenta en el trabajo de Vázquez et al. (2010), que reporta una plataforma para la enseñanza de redes de sensores inalámbricos, que facilita la experiencia directa del alumno con la aplicación práctica de la teoría y por lo tanto mejora su aprendizaje. La plataforma de laboratorio, que se presenta en este trabajo como una solución al problema de infraestructura para el uso de las TIC en el aula de clase, reúne las principales características de las soluciones anteriormente descritas: Bajo costo, uso de los servicios cliente/servidor institucionales y desarrollo de una aplicación práctica de redes de sensores inalámbricos.

La plataforma diseñada usa el protocolo ZigBee. El núcleo central de la plataforma es el sistema embebido de comunicación inalámbrica con adquisición de datos analógicos y digitales en tiempo real, los cuales se registran y muestran al usuario a través de una interface gráfica tipo: Human Machine Interface (HMI). La conectividad inalámbrica desde la red en protocolo ZigBee hacia la red LAN se obtiene configurando la interfaz HMI para ser utilizada de forma remota a través de la red LAN de la comunidad universitaria, por medio de una página web que ha sido creada usando la herramienta 'Web Publishing Tool' del entorno de programación de LabVIEW (Bitter et al., 2007).

Para acceder al uso de la plataforma, el estudiante tiene a su disposición una página web con información introductoria, descrip- 
ción de proyectos realizados por sus compañeros en el área y las guías detalladas de cada práctica de laboratorio. Las guías han sido enfocadas a estudiantes de pregrado en ingeniería en el área de las telecomunicaciones y diseñadas para optimizar la adquisición de habilidades que les permitirán plantear soluciones de comunicación inalámbrica en variadas aplicaciones.

El artículo se organiza como sigue: La sección 2 presenta la descripción de la metodología utilizada en el diseño de la plataforma, la descripción del protocolo ZigBee y de los tres pilares en los que se basa la plataforma desarrollada. Las secciones 3 y 4 presentan los resultados y las conclusiones, respectivamente.

\section{DISEÑO E IMPLEMENTACIÓN DE LA PLATAFORMA DE APRENDIZAJE ZigBee}

El diseño de la plataforma de laboratorio comienza por la definición de las características que facilitarán el logro de los objetivos de aprendizaje, como sigue a continuación: La plataforma de comunicación inalámbrica debe constar de un sistema embebido de fácil reconfiguración que permita la programación de diferentes aplicaciones y de apariencia amigable, en la cual se identifiquen con facilidad todas sus partes. Debe contar con entradas y salidas analógicas y digitales, que permitan monitorear y controlar diferentes tipos de variables. Se debe contar con una interfaz tipo HMI en donde el estudiante pueda realizar el seguimiento del comportamiento de las señales en tiempo real. También debe proveer las facilidades para desarrollar ejemplos de servicios cliente/servidor, que se implementan aprovechando los recursos del router inalámbrico utilizado en la red de la comunidad universitaria.

El siguiente paso en el diseño de la plataforma de aprendizaje, es la selección del protocolo de comunicación inalámbrica a utilizar y las funciones detalladas que debe cumplir el sistema embebido. Con estas funciones en mente se procede al diseño del sistema embebido y los sistemas interconectados, seleccionando los dispositivos y sensores a utilizar para las características de funcionamiento deseadas. Luego se realiza el montaje y pruebas de los dispositi- 
vos y por último la programación de la interfaz HMI y su interconexión con la red LAN.

Para la comunicación inalámbrica se utiliza el protocolo ZigBee, ya que es adecuado para condiciones de bajo consumo energético y de operación en lugares de difícil accesibilidad, además de ser muy utilizado en la construcción de redes inalámbricas para automatización, sistemas de seguridad, redes industriales de control, teleoperación y telemedición, entre otras, (Palanisamy, 2011).

Los módulos XBee son una tecnología que acoge el protocolo ZigBee, basado en el estándar de la IEEE 802.15.4 (Institution of Electrical and Electronics Engineering), estos módulos manejan el protocolo de comunicación ZigBee y proveen dos modos de comunicación: Modo de transmisión serial transparente (modo AT) y el modo Application Programming Interface (modo API) (Zulkifli, 2012).

En este trabajo la plataforma de aprendizaje se desarrolla a partir de los módulos XBee. La programación de la HMI para la medición y análisis de señales en tiempo real se realiza en el entorno de LabVIEW. La plataforma de aprendizaje llevada a cabo en la Universidad Nacional de Colombia - Sede Medellín para el área de Telecomunicaciones está basada en tres pilares (ver Fig. 1), que soportan la estructura de este sistema de laboratorios a bajo costo.

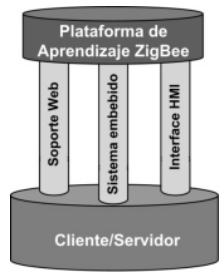

Fig. 1. Plataforma de Aprendizaje ZigBee. Fuente: Autores

\subsection{Soporte Web}

La primera columna, consiste en la interacción con la web, mediante videos de aplicaciones y tutoriales sobre proyectos en desarrollo (Fig. 2). Entre los ejemplos de aplicaciones que se tienen en la página web (www.plataformasZigBee.blogspot.com), se encuentran, el control de posición de un motor DC, una aplicación de 
visión artificial usando $M A T L A B$, la adquisición de datos a través de LabVIEW y el sistema domótico on/off sobre electrodomésticos. Estos ejemplos buscan despertar en los estudiantes el interés por el área de la comunicación inalámbrica para desarrollar nuevas aplicaciones del protocolo ZigBee.

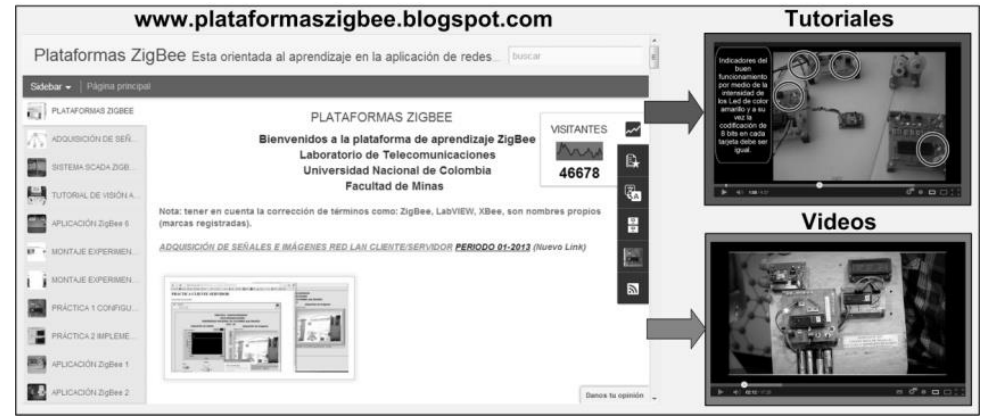

Fig. 2. Plataforma de Aprendizaje ZigBee herramientas Web. Fuente: Autores

\subsection{Sistema Embebido}

La segunda columna de la plataforma, es el diseño del sistema embebido (ver Fig. 3a), que consiste en una tarjeta electrónica con entradas y salidas de señales analógicas y digitales (A/D), las cuales se interpretan bajo un microcontrolador, que se puede programar si es necesario para modificar el propósito de la aplicación con facilidad, es decir para la realización de cambios a los laboratorios y prácticas (ver Fig. 3b).

La simplicidad en la configuración del sistema embebido, tanto en el hardware como el software, dinamiza la implementación de nuevas aplicaciones (Sung, 2011), lo que representa una mejora frente a plataformas estáticas que son destinadas para aplicaciones específicas. Es de resaltar que la principal ventaja de este soporte estructural para las plataformas de aprendizaje ZigBee, consiste en la fácil programación del sistema embebido. 


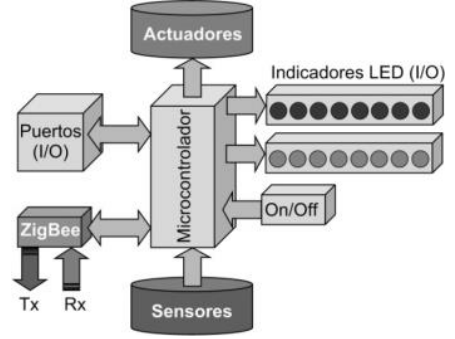

a)

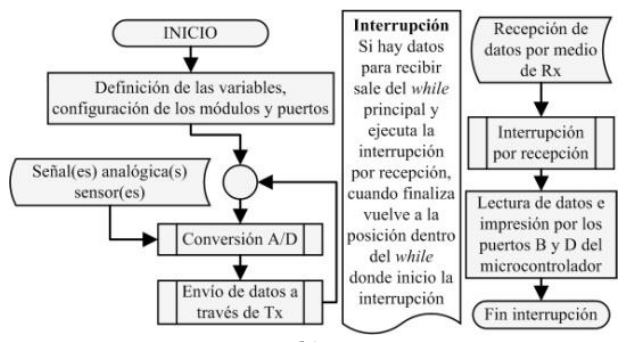

b)

Fig. 3. Estructura del sistema embebido, a) Hardware, b) Software. Fuente: Autores

La tarjeta implementada permite al estudiante interactuar con el sistema de una manera amigable, ya que puede identificar fácilmente cada uno de sus componentes (Fig. 4).

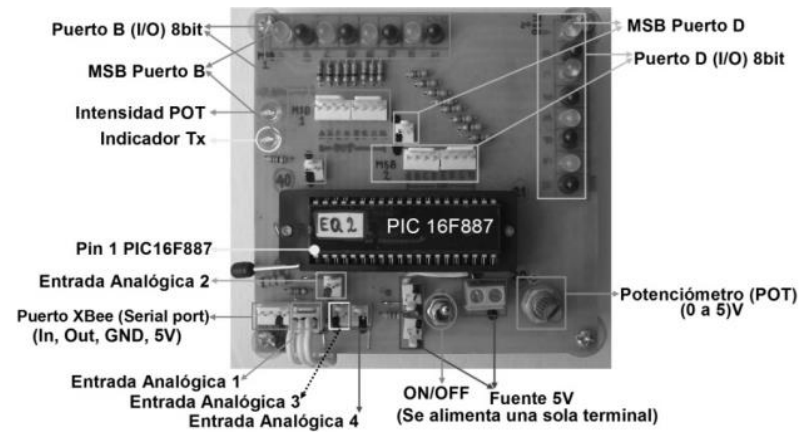

Fig. 4. Componentes de la tarjeta del sistema embebido. Fuente: Autores

Los tres sistemas interconectados consisten en: La transmisión de las señales analógicas de (T) temperatura $(0 \text { a } 50)^{\circ} \mathrm{C}$ a través del sensor LM35 y nivel de (V) voltaje DC (0 a 5)V asociado al potenciómetro, La transmisión de la señal analógica de nivel de voltaje $\mathrm{DC}(0$ a 5$) \mathrm{V}$ asociado al potenciómetro y la recepción de activación en el encendido de 8 LED indicadores conectados a cuatro relevos (110 VAC); y la transmisión de la señal analógica intensidad (L) luminosa del ambiente ( 0 a 100)\% a través de la fotoresistencia y la recepción en el encendido de 8 LED indicadores, conectados a cuatro relevos como señal de salida (ver Fig. 5). 


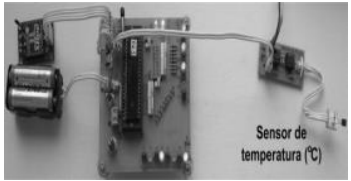

a)

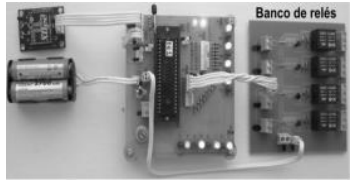

b)

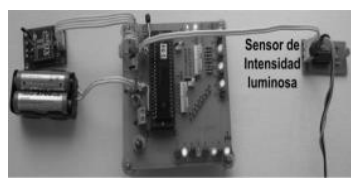

c)

Fig. 5. Sistema interconectado. a) Medición de variables analógicas (T y V), b) Actuador relé a 110VAC, c) Medición de variables analógicas (L). Fuente: Autores

\subsection{Interfaces de usuario}

La tercera columna de esta propuesta, es la integración visual, donde el estudiante tiene acceso a lo que realmente pasa con las señales procesadas, para una aplicación en específico y desde allí realiza el seguimiento del comportamiento de las señales en tiempo real, proveniente del sensor ó actuador de las variables del proceso.

\subsubsection{Interface X-CTU}

En la Fig. 6 se ilustra la interface $X$-CTU herramienta inicial que facilita el entendimiento de las posibilidades de configuración y el tipo de funcionamiento de los módulos XBee. Además en esta interface se puede realizar un test de transmisión de datos (letra azul) y recepción de datos (letra roja), en este caso el equipo 1 envía "Hola equipo 2" y el equipo 2 responde "Hola equipo 1", de esta forma se establece la comunicación escrita de manera instantánea (Chat), en la cual se presentan dos tipos de códigos: En la columna de la izquierda el código ASCII (American Standard Code for Information Interchange) y en la columna de la derecha el código Hexadecimal, (Digi International Inc., 2012).

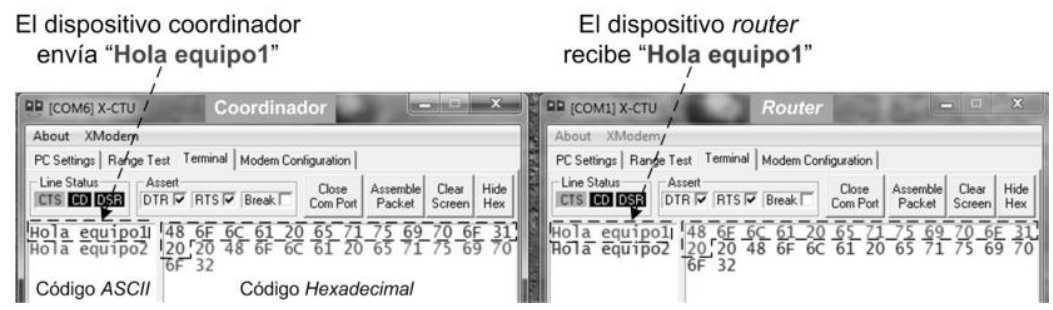

Fig. 6. Interface de configuración X-CTU. Fuente: Autores 


\subsubsection{Interface HMI}

La implementación de la interface gráfica en LabVIEW, se puede observar en la Fig. 7 (a) en el entorno de programación en lenguaje G. En esta se utiliza un componente de comunicación serial denominado VISA (Virtual Instrument Software Architecture), que permite configurar el puerto de comunicación serial (Bitter, 2007). En la Fig. 7 (b) se puede observar el panel frontal de la interface gráfica HMI, en el cual se registran gráficamente las señales analógicas y digitales de entrada y salida; y se realiza la configuración del puerto COM (puerto de enlace serial), así como el establecimiento de la velocidad en baudios. De esta manera el flujo del programa en LabVIEW se puede determinar mediante la representación de la Fig. 8.

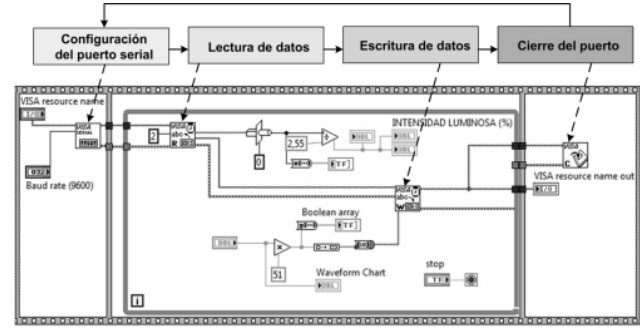

a)

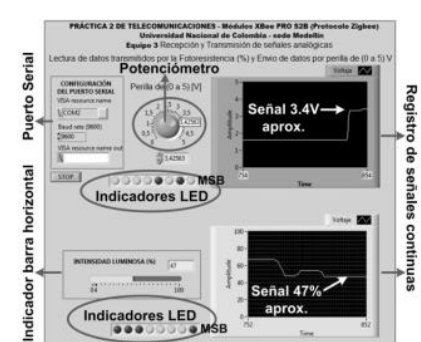

b)

Fig. 7. Entorno de programación LabVIEW/ZigBee. a) Diagrama de bloques, b) Panel frontal (HMI). Fuente: Autores

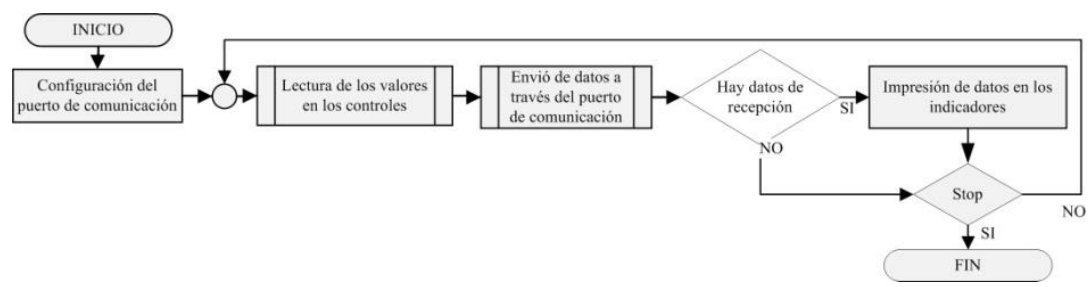

Fig. 8. Flujo de programación en LabVIEW

\subsubsection{Interface cliente/servidor}

Uno de las ventajas que se pueden aprovechar de la red LAN, es la aplicación de servicios cliente/servidor, que se implementan aprovechando los recursos del router inalámbrico utilizado en la red de la comunidad universitaria. Este router es la puerta de 
enlace para replicar la información de la interface en los otros computadores, a través de la página web que ha sido creada usando la herramienta 'Web Publishing Tool' del entorno de programación de LabVIEW. La configuración de la aplicación cliente/servidor para los servicios remotos de la HMI requiere de la dirección IP (internet protocol), la dirección de la puerta de enlace (Gateway) y la máscara de la subred, información que se obtiene de la ventana de comandos de Windows (cmd.exe) ingresando el comando ipconfig (C: $\backslash \ldots . . . . \backslash$ ipconfig). De esta manera se obtiene la conectividad inalámbrica desde una red en protocolo ZigBee hacia la red LAN que se puede observar en la Fig. 9.

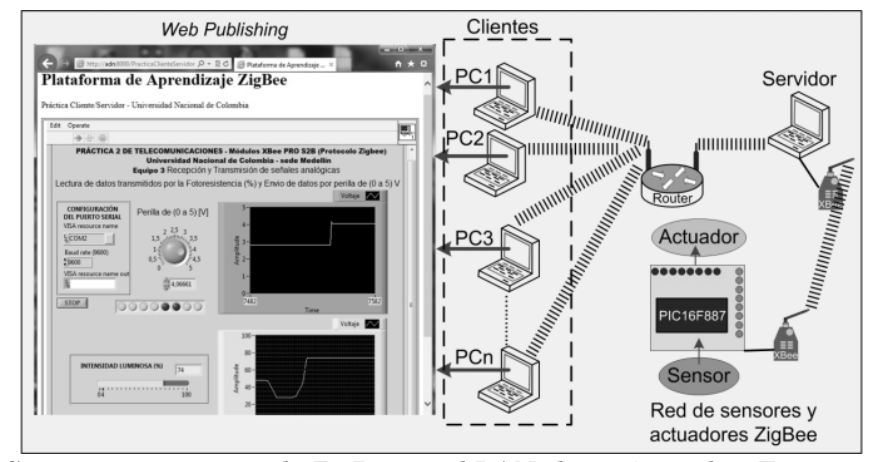

Fig. 9. Sistema interconectado ZigBee - red LAN cliente/servidor. Fuente: Autores

\section{RESULTADOS}

En este momento se cuenta con cuatro de estas plataformas para la realización de prácticas de telecomunicaciones en el laboratorio. Después de la inclusión de estas prácticas en el laboratorio, se ha observado una tendencia en aumento, a presentar proyectos en los que se usa la comunicación inalámbrica por parte de los alumnos.

La plataforma web de aprendizaje ZigBee lleva los registros del número de visitantes que recibe, para establecer su importancia como medio de consulta sobre este tipo de aplicaciones. Desde su implementación en mayo de 2012 cuenta con más de 68 mil visitas, de las cuales alrededor de 40 mil son de fuera del país (datos toma- 
dos de las estadísticas directas de la página: www.plataformasZigBee.blogspot.com, el 23 de septiembre de 2013), con lo que se evidencia un primer impacto a nivel internacional de la propuesta educativa.

La primera práctica propone el establecimiento de la comunicación entre dos computadores portátiles a través de la red inalámbrica ZigBee. Los estudiantes deben conectar los módulos XBee a sus computadores y configurar la red inalámbrica con ayuda de la interface X-CTU. Esta experiencia ha permitido complementar el tratamiento teórico de las redes inalámbricas ya que los estudiantes pasan de la teoría a la práctica entendiendo la importancia de parámetros tales como la velocidad en baudios, el correcto direccionamiento de los elementos que se intercomunican y los canales utilizados. Además de la visualización y trabajo con las tramas de comunicación en código Hexadecimal y en cogido ASCII. En la segunda práctica los estudiantes deben configurar el sistema embebido y los sistemas interconectados, utilizando la interface HMI del LabVIEW, de tal manera que puedan adquirir datos y enviar comandos de manera inalámbrica. Lo que les ha permitido llevar a la práctica los conocimientos de comunicación serial, monitoreo en tiempo real y control inalámbrico.

La tercera práctica permitió a los estudiantes aumentar el alcance de la transmisión de la información utilizando la red LAN universitaria. Esta práctica permite aplicar el conocimiento básico de redes LAN, direccionamiento de internet y configuración de páginas web desde la herramienta adquisición de datos diseñada en LabVIEW. Los estudiantes lograron comunicar sus computadores, monitorear los sistemas interconectados (Fig. 5) y ejecutar comandos de control sobre los mismos, a través de la conectividad que proporciona la red LAN, ampliando así las distancias de comunicación, control y monitoreo inalámbrico. Se logró evidenciar por tanto, que la cobertura de la red inalámbrica ZigBee puede ampliarse hasta el límite de cobertura de la red LAN universitaria. 


\section{CONCLUSIONES}

La integración de la tecnología de las comunicaciones (TIC) en la educación, es un aporte fundamental para el desarrollo de las habilidades y competencias. Con este objetivo en mente se desarrollaron las plataformas de laboratorio presentadas para ser utilizadas en metodologías activas dentro del aula de clase. De acuerdo al trabajo realizado, la topología de la red ZigBee, se puede extender a otras aplicaciones por medio del diseño de plantas y procesos industriales a escala, o directamente al manejo de máquinas de forma remota mediante las redes inalámbricas, haciendo uso de las ventajas que ofrece una HMI y el servicio cliente/servidor implementado en la Internet, con una puerta de enlace que lo lleva a la nube de la información y de esta forma entra por completo a la autopista de la información.

Para un trabajo futuro se podría acondicionar un servidor fijo en el laboratorio de telecomunicaciones, que protocolice de manera adecuada la transmisión y recepción de paquetes interconectados en tiempo real entre las aplicaciones fijas del laboratorio y la página web de plataformas ZigBee. De esta manera se podrían realizar las prácticas a través de la web en modo semipresencial o con interacción en línea y así aumentar la cobertura en número de estudiantes beneficiados con la realización de las prácticas.

\section{AGRADECIMIENTOS}

Este trabajo ha sido desarrollado en el Departamento de Energía Eléctrica y Automática de la Universidad Nacional de Colombia - Sede Medellín, dentro de GAUNAL: Grupo de Automática de la Universidad Nacional, bajo el proyecto MPPT Vectorial.

\section{REFERENCIAS}

Bitter, R., Mohiuddin, T., Nawrocki, M. (2007). LabVIEW Advanced Programming Techniques. (2da. Ed.) Nueva YorK: CRC Press. 
Digi International Inc. (2012). Manual XBee ${ }^{\circledR} /$ XBee-PRO® ZB RF Modules. Recuperado el 10 de julio de 2011, de http://ftp1.digi.com/support/documentation/90000976_K.pdf

Hashemian, R., Pearson, T. R. (2009). A low-cost server-client methodology for remote laboratory access for hardware design. IEEE Frointies in Education Conference, FIE 09-39, 1-5.

Knezek, G.,Christensen, R. (2008). International handbook of information technology in primary and secondary education: The importance of information technology attitudes and competencies in primary and secondary education. Nueva York: Springer.

Sung, W., Hsu, Y. C. (2011). Designing an industrial real-time measurement and monitoring system based on embedded system and ZigBee. Elsevier, Expert Systems with Applications, 38(4), 45224529.UNESCO. (2008). ICT Competency Standards for Teachers. Paris: METIA.

Vazquez, A., Garcia, M., Gay J. A., Cuiñas, I. (2010). Platform for teaching of location technologies based on ZigBee Wireless Sensor Networks by learning through-play theory. IEEE Education Engineering (EDUCON), 1299-1305.

Velásquez, M. A., Lopéz, R. (2008). Una mirada crítica al papel de las TIC en la educación superior en Colombia. Revista E-mail Educativo. Recuperado el 10 de septiembre de 2012, de http://www.revistas.unal.edu.co/index.php/email/article/view/12623

Zulkifli, N., Harun, F., Azahar, N. (2012). XBee wireless sensor network for Heart Rate Monitoring in sport training. IEEE Conference Biomedical Enginnering (ICoBE). 